\title{
Intervention Protocols for the Treatment of Metabolic Syndrome and its Components in Children and Adolescents: A Systematic Review
}

\author{
Ewerton Eiti Yamada ${ }^{1}$, Mirian Ueda Yamaguchi ${ }^{2 *}$ and Marcelo Picinin \\ Bernuci $^{2}$ \\ ${ }^{1}$ Departamento de Fisiologia do Exercício, Universidade Cesumar (UniCesumar), \\ Maringá, Paraná, Brasil \\ ${ }^{2}$ Departamento de Promoção de Saúde, Universidade Cesumar (UniCesumar), \\ Instituto Cesumar de Ciência, Tecnologia e Inovação (ICETI), Maringá, Paraná, \\ Brasil \\ *Corresponding Author: Marcelo Picinin Bernuci, Departamento de Promoção de \\ Saúde, Universidade Cesumar (UniCesumar), Instituto Cesumar de Ciência, \\ Tecnologia e Inovação (ICETI), Maringá, Paraná, Brasil.
}

Received: February 20, 2021

Published: March 20, 2021

(C) All rights are reserved by Mirian Ueda

Yamaguchi., et al.

\begin{abstract}
Physical exercise, in general, promotes the improvement of several metabolic markers in overweight and obese children and adolescents, however, the evidence still presents some divergences regarding the impact of different training protocols on metabolic syndrome (MS). This systematic review aimed to assess and discuss the impact of different physical exercise protocols on the prevalence of MS and its markers in children and adolescents. The study followed the guidelines proposed by PRISMA-E 2012 and searches for articles were performed in the electronic database PubMed. As an inclusion criterion, the articles should explore the intervention and the impact of physical exercise on MS and its components on children and adolescents. A total of 348 articles were found and, after applying the eligibility criteria, 10 studies remained for qualitative analysis. Of the 10 selected studies, 3 used aerobic training protocol, 4 applied concurrent training, 2 recreational interventions and only 1 resisted training. Regardless of the protocol, physical exercise is a fundamental tool in maintaining several blood biomarkers and body composition, although protocols with aerobic exercises have shown good results. However, further investigations are still needed.
\end{abstract}

Keywords: Metabolic Syndrome; Children and Adolescents; Physical Exercise

\section{Introduction}

Studies have shown a strong association between obesity and other comorbidities such as insulin resistance, dyslipidemia and arterial hypertension, which, consequently, increase the risks of Cardiovascular Diseases (CVD) [1]. This set of cardiovascular risk factors, of metabolic origin, when occurring/developed simultaneously, is known as Metabolic Syndrome (MetS) [2]. Data indicate that people with MS have a risk of CVD and CVD mortality increased by two or more times, in comparison to people without the syndrome [3]. This data is alarming, since cardiovascular diseases are identified as the chronic non-communicable diseases (NCDs) that kill the most people in the world. According to the World Health Statistics published by the World Health Organization in 2020, it is estimated that in 2016 approximately 17.9 million people lost their lives due to cardiovascular diseases, a number that represents $31 \%$ of all deaths that occurred in the year [4].

Due to the strong association between obesity and the development of MS, the early occurrence of overweight in the young population is of great concern. The prevalence of childhood obesity has 
increased at a significant pace in recent years and is considered one of the greatest challenges to public health worldwide in the 21st century. According to data published by the World Obesity Federation (WOF) in the Childhood Obesity Atlas, in 2019, about 150 million children and adolescents around the world were considered obese, and that number is expected to reach 250 million by 2030 [5]. Together and not only excluded to the adult population, the prevalence of MS has also increased as higher rates of obesity in children and adolescents are identified [6], a scenario that may be more serious in view of the difficulties in carrying out early diagnosis of MS.

In fact, establishing criteria for the diagnosis of MS in childhood and adolescence has been a challenge because some components of the syndrome may vary according to the individual's age group and, therefore, there are still some divergences in the criteria for its definition. These divergences have made it difficult to accurately estimate the prevalence of MS in this population and may underestimate the number of cases [7]. However, regardless of the criteria used in the definition, overweight and obese children are at increased risk of developing future metabolic and cardiovascular diseases, as well as increase in premature mortality in adulthood [8], which has made this population the focus of global public health strategies. Thus, the high prevalence of childhood obesity, MS and future CVD risk is worrisome and should alert professionals who care for children and adolescents, as early intervention in the child's lifestyle is extremely important in order to reduce the incidence of these disorders and ensure a healthier adult life [9].

Admittedly, low levels of physical activity and aerobic fitness are associated with greater risks of MS and, therefore, physical exercise has a fundamental role in the treatment and prevention of MS [10]. Studies that investigated the impact of physical exercise on the components of MS concluded that its practice has positive results on metabolic parameters, reduces blood pressure and insulin resistance, in addition to improving well-being and the predisposition to maintain physical activity in adulthood, guaranteeing lower risks of cardiovascular diseases and longer life expectancy [11]. Although the practice of physical exercise promotes improvement of several metabolic markers in overweight and obese children and adolescents [12], the evidence still presents some divergences regarding the impact of different training protocols on obesity and markers metabolic [13]. In this sense, the objective of this systematic review was to assess and discuss the impact of different physical exercise protocols on the prevalence of MS and its markers in children and adolescents.

\section{Materials and Methods}

The present study is a systematic review, developed according to the guidelines proposed by PRISMA-E 2012 (Preferred Reporting Items for Systematic Reviews and Meta-Analyzes) [14].

\section{Search and source of information}

Searches for articles were carried out in the electronic database PubMed Central - PMC (https://www.ncbi.nlm.nih.gov/pmc/), between December 2019 and January 2020. To perform the search, we used as the main term "Metabolic Syndrome", associated with secondary terms "Children and Adolescents" and "Physical Exercise".

\section{Eligebility criteria}

As an inclusion criterion, it was defined that the articles should have an experimental design of an interventional character, involving obese or overweight children and adolescents, with characteristics of MS. In addition, studies should mandatorily explore the intervention and impact of physical exercise on the prevalence of MS and/or its components (obesity, dyslipidemia, glycemia and insulin resistance, blood pressure), with or without multidisciplinary intervention (nutritional) and psychological), as long as there was no use of medications, in order to ensure that the results were not influenced by other therapies, other than behavioral

Studies involving adults were excluded, which investigated only the relationship between Physical Fitness and MS and its components, incidence, pathophysiology/pathophysiology. No dates and language limits were applied. Systematic reviews and meta-analyzes were also not included in the data extraction.

\section{Data extraction}

After screening, eligibility and inclusion of the studies, the extraction of the relevant data was performed and stored in an Excel spreadsheet. 


\section{Results}

\section{Study selection}

After defining the search strategies, a total of 348 articles were found in the PubMed database. Among these, 265 were excluded due to the irrelevance of the title and abstract, leaving 83 articles. After applying the eligibility criteria, 73 were eliminated for not meeting the requirements, leaving 10 for qualitative analysis. The selection process is detailed in figure 1.

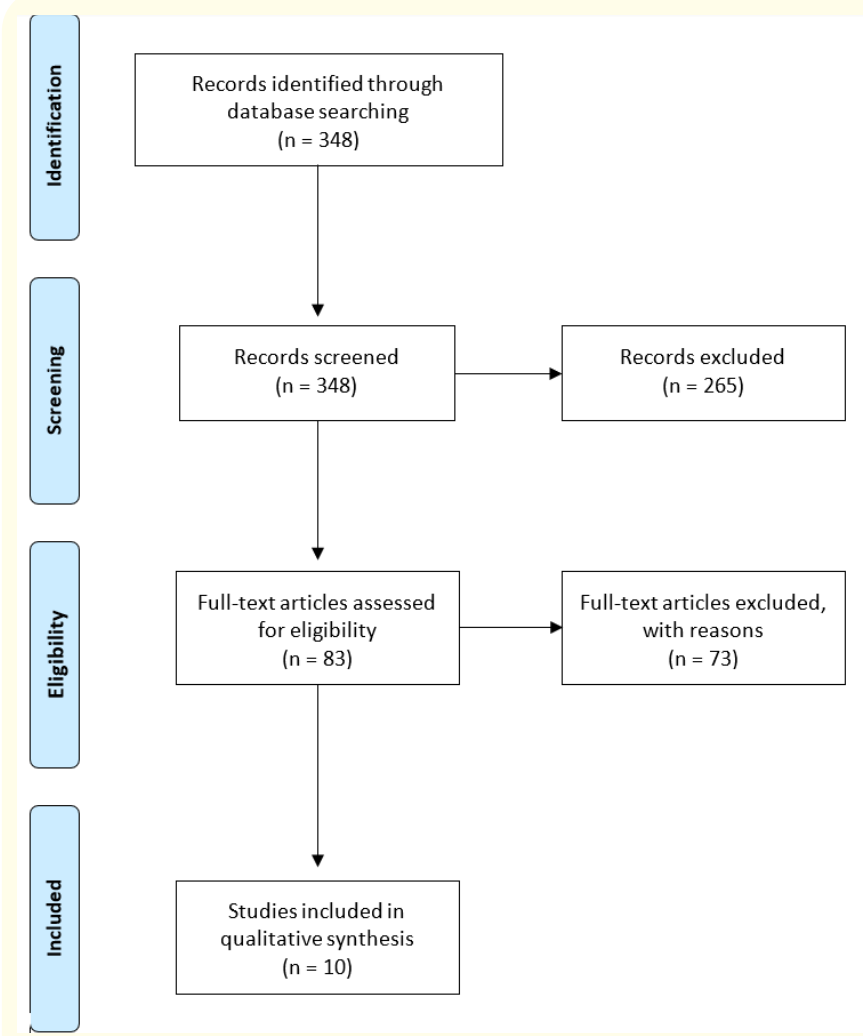

Figure 1: Diagram of the flow of identification, screening, eligibility and inclusion of articles [14].

The description of the studies, detailing the population, the intervention and the outcomes are presented in tables 1, 2, 3 and 4, and were divided according to the exercise protocol used.

\section{Concurrent training and metabolic syndrome markers}

After reading, four studies were selected that fit the concurrent training model, as shown in Table 1 . All four studies had interventions by nutritionists and psychologists, in addition to physical exercise, however, there was no standardization in frequency, duration and intensity training.

The findings showed a significant impact on obesity, metabolic and cardiometabolic risk markers. Although everyone evaluated the effect of the intervention on MS markers, Bluher and colleagues were the only ones who did not show the prevalence of the syndrome [15]. In the others, with the exception of Wickham and colleagues, which, in contrast, showed an increase in prevalence, there was a reduction in the number of subjects with MS by half, approximately, at the end of the intervention [16].

Significant reductions in BMI and fat percentage, as well as improvements in the lipid profile, represented by a reduction in LDL and total cholesterol levels, were present in all studies. Only Masquio and colleagues found increases in HDL [17], while reductions in triglycerides found by Bustos and colleagues [18]. As for glucose metabolism and insulin resistance, improvements were presented in the work of Bluher and colleagues [15] through the OGTT and HbA1c curve tests, Bustos and colleagues [18] by measuring blood glucose and Masquio and colleagues [17] through HOMA-IR and QUICKI. Finally, Bustos and colleagues [18] and Masquio and colleagues [17] observed a decrease in systolic and diastolic blood pressure.

\section{Resistance training and metabolic syndrome markers}

In the search, only Vásquez and colleagues [19] submitted their sample to a resistance training model - table 2. The intervention also had nutritional and psychological assistance.

The results showed that resistance training can reduce the prevalence of MS, improve body composition and physical condition. After 6 and 9 months of interruption of the intervention, all markers returned to baseline, suggesting that, in order to maintain 


\begin{tabular}{|c|c|c|c|c|c|c|c|}
\hline \multirow[b]{2}{*}{ Reference } & \multirow[b]{2}{*}{ Population } & \multicolumn{4}{|c|}{ Intervention } & \multirow[t]{2}{*}{ Results } & \multirow[t]{2}{*}{ Conclusion } \\
\hline & & Protocol & Intensity & $\begin{array}{l}\text { Program } \\
\text { duration }\end{array}$ & $\begin{array}{c}\text { other accom- } \\
\text { paniments }\end{array}$ & & \\
\hline $\begin{array}{c}\text { BLUHER., } \\
\text { et al. } \\
2013 \text { [15] }\end{array}$ & $\begin{array}{c}\mathrm{N}=115 \\
7-18 \text { years }\end{array}$ & $\begin{array}{l}\text { - Concurrent } \\
\text { Training } \\
\text {-150min / week } \\
\text { (90 min supervised } \\
+60 \text { min free) }\end{array}$ & - & $\begin{array}{c}12 \\
\text { months }\end{array}$ & $\begin{array}{l}\text { Nutritional and } \\
\text { psychological } \\
\text { guidance }\end{array}$ & $\begin{array}{c}\downarrow \text { PC; } \downarrow \text { BMI; } \downarrow \text { CA; } \downarrow \\
\text { Waist / height ratio; } \\
\downarrow \% \text { GC; } \downarrow \text { AGL; } \downarrow \text { GGT; } \\
\downarrow \text { OGTT curve; } \downarrow \\
\text { HbA1c }\end{array}$ & \begin{tabular}{|} 
The combined \\
exercise / lifestyle \\
program KLAKS \\
significantly improves \\
the markers of obesity \\
and glycemic control. \\
Impaired cardiome- \\
tabolic risk markers \\
are also favorably \\
influenced.
\end{tabular} \\
\hline \begin{tabular}{|} 
BUSTOS., \\
et al. 2015 \\
{$[18]$}
\end{tabular} & $\begin{array}{c}N=28 \\
(10 \text { with } S M) \\
9,5 \pm 2 \text { years }\end{array}$ & $\begin{array}{c}\text { - Concurrent } \\
\text { training } \\
\text { •50min / day; } 2 \mathrm{x} / \\
\text { week }\end{array}$ & - & $\begin{array}{c}8 \\
\text { months }\end{array}$ & $\begin{array}{l}\text { Nutritional and } \\
\text { psychological } \\
\text { guidance }\end{array}$ & $\begin{array}{c}\downarrow \text { BMI; } \downarrow \text { CA } \downarrow \% \text { GC; } \\
\downarrow \text { PAS and PAD; } \downarrow \\
\text { Blood glucose; } \downarrow \text { CT; } \\
\downarrow \text { LDL; } \downarrow \text { Triglyceri- } \\
\text { des; } \\
\\
\text { Of the } 10 \text { that were } \\
\text { classified with MS, } \\
\text { only } 5 \text { were still clas- } \\
\text { sified with MS after. }\end{array}$ & \begin{tabular}{|} 
The Bright Bodies \\
Program produced \\
significant and \\
positive changes in the \\
anthropometric and \\
metabolic param- \\
eters in this group of \\
children.
\end{tabular} \\
\hline
\end{tabular}

Table 1: Summaries of individual data showing the interventions and outcomes of concurrent training on MS markers.

BMI - Body Mass Index; \% GC - Body Fat Percentage; LDL - Low Density Lipoprotein; CT - Total Cholesterol; CA - Abdominal circumference; SBP - Systolic Blood Pressure; DBP - Diastolic Blood Pressure; AGL - Free Fatty Acid; PC - Body Weight; OGTT - Oral Glucose Tolerance Test; GGT - glutamyl transferase range 
the results obtained, the sustainability of the practice of physical exercise is necessary. A reduction in BMI z-score, waist circumference was observed, while improvements in the percentage of fat occurred only in girls. In a distance traveled test, there was also improvement. Although the authors found a reduction in the prevalence of MS, data regarding the components of MS (blood glucose, triglycerides, HDL and blood pressure) were not exposed.
Aerobic training and metabolic syndrome markers

Three studies applied the aerobic training model, as detailed in table 3. The surveys had no other interventions than aerobic exercise. All authors used heart rate as an intensity control parameter, however, each following a specific protocol for intensity, duration and frequency of weekly training.

\begin{tabular}{|c|c|c|c|c|c|c|c|}
\hline \multirow[b]{2}{*}{ Reference } & \multirow[b]{2}{*}{ Population } & \multicolumn{4}{|c|}{ Intervention } & \multirow[t]{2}{*}{ Results } & \multirow[t]{2}{*}{ Conclusion } \\
\hline & & Protocol & Intensity & $\begin{array}{l}\text { Program } \\
\text { duration }\end{array}$ & $\begin{array}{c}\text { Other } \\
\text { follow-ups }\end{array}$ & & \\
\hline
\end{tabular}

Table 2: Summary of individual data presenting the interventions and outcomes of resistance training on mS markers. zBMI - Body Mass Index z-score; \%GC - Body FatPercentage; CA - Abdominal Circumference.

In general, the results showed that aerobic training performed at the heart rate corresponding to the zone of greater fat oxidation significantly reduces obesity markers and improves the components of MS.

As a marker of central obesity, waist circumference showed a significant reduction in the three studies and, in two, there were improvements in weight and BMI. Reductions in glycemic and triglyceride levels were also present in all studies. On the other hand, only two find increases in HDL. Blood pressure was assessed by two studies, however, only Ben Ounis and colleagues [20] found significant improvements.

\section{Playful training}

Finally, two studies applied training in a playful way - table 4 . In this session, works that used games, sports and school physical education as an exercise mode were included, ensuring that the intervention was, for the most part, playful. Both were accompanied by nutritional guidance.
Only one of the studies showed the prevalence of MS, however, both showed significance in the results regarding metabolic and anthropometric markers. Both Chen and colleagues [21], and Nascimento and colleagues [22] observed improvements in BMI, body fat percentage, HOMA-IR index, insulin, LDL and total cholesterol.

Significant reductions in waist circumference, blood pressure and triglycerides were observed only in the work by Chen and colleagues [21].

\section{Discussion}

The present study aimed to assess and discuss the impact of different physical exercise protocols on the prevalence of MS and/ or its markers in children and adolescents. In general, the results presented here show the importance of physical exercise as a nonpharmacological strategy to reduce the prevalence of MS and its impact both on anthropometric parameters and on the cardiometabolic health of children and adolescents. Although the focus of the analyzes was on the influence of different physical exercise 


\begin{tabular}{|c|c|c|c|c|c|c|c|}
\hline Reference & Population & \multicolumn{4}{|c|}{ Intervention } & Results & Conclusion \\
\hline $\begin{array}{l}\text { NASCI- } \\
\text { MENT., et } \\
\text { al. } 2014\end{array}$ & $\begin{array}{c}\text { Longitudinal } \\
\mathrm{N}=575-18 \\
\text { years }\end{array}$ & $\begin{array}{l}\text { - } 3 \mathrm{~h} \text { of school physical } \\
\text { education• } 2 \text { sessions } \\
\text { (1h each)of training, } \\
\text { aerobic circuit, strength } \\
\text { training, coordination } \\
\text { and balance, games and } \\
\text { longing• total 5h per } \\
\text { week }\end{array}$ & $\begin{array}{l}\text { 80\% of } \\
\text { FcMáx }\end{array}$ & 8 months & $\begin{array}{l}\text { Nutritional } \\
\text { guidance }\end{array}$ & $\begin{array}{c}\bullet \text { BMI; } \bullet \text { CT; LDL; } \bullet \\
\text { Insulin; } \bullet \text { HOMA-IR; } \\
\% \text { GC; } \uparrow \text { Ratio HDL/CT. } \\
\text { Improvements in BMI } \\
\text { prevented the reduction } \\
\text { of adiponectin and cor- } \\
\text { related with increases in } \\
\text { HMW and MMW }\end{array}$ & $\begin{array}{l}\text { The increase related to } \\
\text { obesity in mS characte- } \\
\text { ristics may be related to } \\
\text { lower adiponectin. HMW } \\
\text { and MMW were the } \\
\text { multimeters that most } \\
\text { explained the resources } \\
\text { of the DM. The interven- } \\
\text { tion program improved } \\
\text { the lipid profile and IR } \\
\text { and prevented the reduc- } \\
\text { tion of adiponectin. }\end{array}$ \\
\hline
\end{tabular}

Table 4: Summary of individual data presenting the interventions and outcomes of mixed playful training on mS markers.

PC -Body Weight; BMI - Body Mass Index; CA - Circumference Abdominal; \%GC - Body Fat Percentage; CT - Total Cholesterol;

LDL - Low Density Lipoprotein; BP - Blood Pressure.

protocols (concurrent, resistance training, aerobic, mixed play) on the prevalence of MS and its impact on clinical markers, the importance of multidisciplinary interventions in this context was also observed. Among the ten studies selected for this review, seven included other follow-ups, with psychologists, nutritionists or both [15-19,21,22], where the majority demonstrated positive results on MS. These data suggest that the multidisciplinary intervention is efficient to improve the MS, however, it must be well monitored in order to avoid failures during the intervention.

\section{Concurrent training}

Concurrent training consists of a model where two physical capacities are worked on in the same training session, for example, aerobic strength and endurance. This training model has been the subject of many discussions due to possible interference that a given stimulus could cause over the other, impairing performance, development of strength, power and muscle mass gain. However, when it comes to improving health in general, the literature has shown that the simultaneous work of aerobic and resistance exercises in the same training session has positively impacted on the components of MS improving the anthropometric markers, lipid profile [25] and glucose metabolism [26]. In the present review, we can see that all studies involving the concurrent training model were significant in improving body mass and composition, reducing BMI and fat percentage, as well as improving lipid profile markers, meeting what literature has shown in populations without the syndrome [25]. As for glucose metabolism and MS prevalence, the findings of this review showed significant reductions, except in the work of Wickham and colleagues [16] in which the results were opposed to the other studies $[15,17,18]$, showing no significant change in blood glucose and an increase in the prevalence of the syndrome. These data lead us to believe that there was a flaw in the study by Wickham and colleagues [16], considering the course and the large discrepancy in the results when compared to the other studies. 
Thus, the set of findings suggests that when well designed and controlled, the concurrent training model can be an effective strategy in the treatment of MS and its components, improving anthropometric markers, glucose metabolism and at least one component of the lipid profile. However, despite these improvements, further investigations and better controls involving this training model in children and adolescents with MS are still needed, as data are still scarce and have been shown to be inconsistent.

\section{Resistance training}

Although the prescription of aerobic exercises to improve health is a common practice, resistance training has become popular and the subject of studies in recent years. Resistance training can be defined as a method that involves the use of resistive loads (free weights, weight machines, elastic ropes, medicine ball, body weight, etc.) aiming at a variety of objectives, such as improving health, improved sports performance, increased muscle mass, strength and others.

In addition to aesthetic improvement and physical-sports performance, studies have shown the importance of maintaining adequate levels of muscle mass, as low levels are associated with increased risk of cardiovascular disease [27] and cardiometabolic risk in adolescents [28], as well as greater risks of type 2 diabetes [29] and prevalence of MS in adults [30]. Thus, we can consider resistance training as an important tool to reduce the chances of cardiovascular events and metabolic disorders, resulting from and characteristic of the syndrome, since this practice is widely used to maintain and develop muscle volume and improve body composition. On the other hand, the impact of resistance exercise on lipid markers seems inconsistent. However, a systematic review has pointed out that resistance training applied at moderate intensity (50-85\% of $1 \mathrm{RM})$ promotes better effects on the lipid profile when compared to higher intensities (> 85\% of 1RM) [31].

Regarding the effect of resistance training on arterial hypertension, there is a lack of studies in this regard. In addition, most studies have included subjects using antihypertensive drugs whose blood pressure is within normal levels, making it difficult to assess the real effect of exercise on blood pressure and finding little significant results. However, Boeno and colleagues [32] when comparing two training models, concluded that both resistance and aerobic training can promote similar reductions in systolic blood pressure.

Although Vásquez and colleagues [19], have not evaluated the gain in muscle mass in their work, observed that resistance training promoted a reduction in body weight, improvements in physical conditioning and a reduction in the prevalence of MS. In addition, they observed that 6 and 9 months after ceasing exercise, the gains obtained regressed close to the baseline, suggesting the need for constant exercise to ensure that the results are sustained. Another limitation of the work by Vásquez and colleagues [19] is due to the fact that they did not show results for blood markers and blood pressure, making it impossible to compare data with other findings. Although the authors indicate resistance training to reduce the prevalence of MS, we emphasize that the works found in the literature are insufficient and there is a need for more research in this sense for a more conclusive result.

\section{Aerobic training}

Aerobic training is characterized by performing rhythmic continuous exercises that involve large muscle groups whose energy production depends, predominantly, on aerobic metabolism, that is, it depends on the presence of oxygen to extract energy in the form of adenosine triphosphate of carbohydrates, fatty acids or amino acids. Aerobic training promotes adaptations in the cardiovascular system, improving the function and size of the heart and arteries, as well as the increase of vessels in the skeletal muscle, reflecting both in the improvement of aerobic power and physical performance, as well as in cardiovascular health [32]. Some authors have shown an inverse association between cardiorespiratory fitness and MS, reinforcing the importance of works that optimize V02Máx [34]). Therefore, the better the cardiorespiratory fitness, the lower the chances of developing MS.

Recently, Houzelle and colleagues [35], also showed that aerobically trained people have improved oxidative capacity and sensitivity to muscle insulin due to adaptations in mitochondrial dynamics, suggesting that the practice of aerobic exercises may act in glycemic control and increase fat oxidation. These findings support the results found in the present systematic review, where we can observe improvements in glycemic control, in the lipid profile, as well as an increase in the fat oxidation rate. 
Although aerobic exercise is often recommended to control blood pressure, only Ben Ounis and colleagues [20] found significant reductions in blood pressure levels. However, we noticed that in other studies, the subjects' blood pressure was already at normal levels, thus not showing any significant reductions.

\section{Playful mixed training}

In the scope of physical exercise, training is understood as an organized and systematic practice aiming at physical improvement, in order to improve the ability to perform tasks that involve motor demands. However, when aimed at children and adolescents, the application of playfulness in training can make the practice more attractive and stimulating, ensuring greater participation by the participants.

Although few studies are performed with games and games, our findings showed the relevance of interventions with games, sports and physical education at school. Chen and colleagues [21] submitted their sample to a protocol based on beach games and sports. After the short-term intervention, the authors showed significant improvements in anthropometric and cardiometabolic measures and a significant reduction in the prevalence of MS. Still with a proposal of a playful character, involving school physical education, Nascimento and colleagues [22] despite not having a prevalence of MS, they showed that school physical education promotes improvement in body composition, lipid profile, metabolism and glucose control. Although we have not found other works in the same direction, we can conclude that an active childhood, even with simple recreational games and school physical education, can reverse metabolic diseases.

One of the limitations of the present review was the difficulty in grouping the results of the different studies for a deeper analysis. The heterogeneity of the diagnostic protocols used, varying from definitions proposed by the International Diabetes Federation - IDF [36] and WHO [37] made it impossible to perform a metaanalysis due to inconsistent results. The lack of consensus on which definition of the syndrome should be applied has been reported in another study [38] and remains a challenge for carrying out more elaborate studies.
A definition frequently used for children and adolescents is proposed by the IDF, in which abdominal obesity is considered a mandatory condition for diagnosis [36]. For the Federation, children under 10 years old cannot be diagnosed with MS, but their family members should be alerted about the state of overweight/obesity. For children over 10 and under 16, the diagnosis is made by the presence of abdominal obesity (percentile $\geq 90$ ) together with two or more clinical changes, such as triglycerides $\geq 150 \mathrm{mg} / \mathrm{dL}$, HDL cholesterol $<40 \mathrm{mg} / \mathrm{dL}$, systolic blood pressure $\geq 130 /$ diastolic $\geq 85$ $\mathrm{mm} \mathrm{Hg}$, plasma glucose $\geq 100 \mathrm{mg} / \mathrm{dL}$ or type 2 diabetes mellitus. For adolescents over 16 years old, the same criteria are used for adults. In view of the vast number of definitions (more than 40) and the lack of consensus between the agencies, the prevalence of MS is underestimated or overestimated, depending on which definition is used. Therefore, in addition to just diagnosing a patient, it is important to individually assess each cardiometabolic and biochemical risk factor related to the syndrome [38].

\section{Conclusion}

Anyway, the outcomes presented here make it evident that, regardless of the protocol, physical exercise is a fundamental tool in the maintenance of various blood biomarkers and body composition and, consequently, this maintenance will positively imply in the improvement of MS in children and adolescents. Furthermore, the importance of an early intervention, in childhood and adolescence, whether multidisciplinary or not, is also recognized, adopting an active lifestyle and healthy eating habits because, if serious measures are not taken, the prevalence of related pathologies obesity will be increasing. Although there is a need for further investigation, especially with resistance training, protocols with aerobic exercises have stood out and shown good results, even without any other type of intervention. However, we know that nutritional and psychological monitoring can be tools of great importance in the treatment process and change of habits.

\section{Acknowledgements}

This research was possible thanks to the financial support of the Instituto Cesumar de Ciência, Tecnologia e Inovação (ICETI), Maringa, Paraná, Brazil.

\section{Conflict of Interest}

The authors declare no conflict of interest. 


\section{Bibliography}

1. Murni I K., et al. "Risk of metabolic syndrome and early vascular markers for atherosclerosis in obese Indonesian adolescents". Pediatrics and International Child Health 40.2 (2020): 1-7.

2. Grundy S., et al. "Definition of metabolic syndrome. NHLBI/ AHA conference proceedings". Circulation 109.27 (2004): 433438.

3. Mottillo S., et al. "The Metabolic Syndrome and Cardiovascular Risk a Systematic Review and Meta-Analysis". Journal of the American College of Cardiology 56.14 (2010): 1113-1132.

4. World Health Organization (WHO). "World Health Statistics 2020: monitoring health for the SDGs, sustainable development goals". Geneva (2020).

5. World Obesity federation (WOF). "Atlas of Childhood Obesity". London (2019).

6. Ogden C L., et al. "Trends in Obesity Prevalence Among Children and Adolescents in the United States, 1988-1994 Through 2013-2014". Journal of the American Medical Association 315.21 (2016): 2292-2299.

7. Al-Hamada and D RAMAN. "Metabolic syndrome in children and adolescents". Translational Pediatrics 4.4 (2017): 397407.

8. Yanovski J A. "Pediatric obesity. An introduction". Appetite 93 (2015): 3-12.

9. Deboer M D. "Assessing and managing the metabolic syndrome in children and adolescents". Nutrients 11.8 (2019): 1-12.

10. Neto A S., et al. "Physical activity, cardiorespiratory fitness, and metabolic syndrome in adolescents: a cross-sectional study". BMC Public Health 11.674 (2011): 1-7.

11. Brandão A P., et al. "Management of metabolic syndrome in young population". American Journal of Therapeutics 15.4 (2008): 356-361.

12. Paes ST., et al. "Efeitos metabólicos do exercício físico na obesidade infantil: uma visão atual". Revista Paulista de Pediatria 33.1 (2015): 122-129.
13. Whooten R., et al. "Physical activity in adolescents and children and relationship to metabolic health". Current Opinion in Endocrinology, Diabetes and Obesity 26.1 (2019): 25-31.

14. Welch., et al. "Extending the PRISMA statement to equity-focused systematic reviews (PRISMA-E 2012): explanation and elaboration". Journal of Clinical Epidemiology 70 (2016): 6889.

15. Blüher S., et al. "The one-year exercise and lifestyle intervention program KLAKS: Effects on anthropometric parameters, cardiometabolic risk factors and glycemic control in childhood obesity". Metabolism 63.3 (2014): 422-430.

16. Wickham E P., et al. "Prevalence of the Metabolic Syndrome Among Obese Adolescents Enrolled in a Multidisciplinary Weight Management Program: Clinical Correlates and Response to Treatment". Metabolic Syndrome and Related Disorders 7.3 (2009): 179-186.

17. Masquio DCL., et al. "The Role of Free Fatty Acids in the Inflammatory and Cardiometabolic Profile in Adolescents With Metabolic Syndrome Engaged in Interdisciplinary Therapy". The Journal of Nutritional Biochemistry 33 (2016): 136-144.

18. Bustos., et al. "Impacto del programa de manejo de la obesidad Bright Bodies aplicado a niños y adolescentes chilenos". Revista Médica de Chile 143.9 (2015): 1136-1143.

19. VÁSQUEZ F., et al. "Efecto residual del ejercicio de fuerza muscular en la prevención secundaria de la obesidad infantil". Nutrición Hospitalaria 28.2 (2013): 333-339.

20. Ben Ounis, O., et al. "Exercise improves the ApoB/ApoA-i ratio, a marker of the metabolic syndrome in obese children". Acta Paediatrica 99.11 (2010): 1679-1685.

21. Chen A K., et al. "Effect of a short-term diet and exercise intervention on metabolic syndrome in overweight children". Metabolism Clinical and Experimental 55.7 (2006): 871-878.

22. Nascimento H., et al. "Adiponectin and Markers of Metabolic Syndrome in Obese Children and Adolescents: Impact of 8-mo Regular Physical Exercise Program". Pediatric Research 76.2 (2014): 159-165. 
23. Kamal N and RAGY M M. "The effects of exercise on C-reactive protein, insulin, leptin and some cardiometabolic risk factors in Egyptian children with or without metabolic syndrome". Diabetology and Metabolic Syndrome 4.27 (2012): 27.

24. Zguira M S., et al. "Effect of an 8-week individualized training program on blood biomarkers, adipokines and endothelial function in obeses young adolescents with and without metabolic syndrome". International Journal of Environmental Research and Public Health 16.5 (2019): 751-763.

25. Antunes B M L., et al. "Effect of concurrent training on genderspecific biochemical variables and adiposity in obese adolescents". Archives of Endocrinology and Metabolism 59.4 (2015): 303-309.

26. Bassi D., et al. "Potential effects on cardiorespiratory and metabolic status after a concurrent strength and endurance training program in diabetes patients - a randomized controlled trial". Sports Medicine 2.31 (2016): 1-13.

27. Srikanthan., et al. "Relation of Muscle Mass and Fat Mass to Cardiovascular Disease Mortality". American Journal of Cardiology 117.8 (2016): 1355-1369.

28. Burrows R., et al. "Low muscle mass is associated with cardiometabolic risk regardless of nutritional status in adolescents: A cross-sectional study in a Chilean birth cohort". Pediatric Diabetes 18.8 (2017): 895-902.

29. Son JW., et al. "Low muscle mass and risk of type 2 diabetes in middle-aged and older adults: Findings from the KoGES". Diabetologia 60.5 (2017): 865-872.

30. Carvalho., et al. "Association between skeletal mass indices and metabolic syndrome in brazilian adults". Journal of Clinical Densitometry: Assessment and Management of Musculoskeletal Health (2020): 1-11.

31. Mann., et al. "Differential Effects of Aerobic Exercise, Resistance Training and Combined Exercise Modalities on Cholesterol and the Lipid Profile: Review, Synthesis and Recommendations". Sports Medicine 44.xx (2014): 211-221.
32. Boeno F P., et al. "Effect of aerobic and resistance exercise training on inflammation, endothelial function and ambulatory blood pressure in middle-aged hypertensive patients". Journal of Hypertension XX.XX (2020): 2501-2509.

33. Hellsten Y and Nyberg M. "Cardiovascula adaptations to exercise training". Comprehensive Physiology 6 (2016): 1-32.

34. Wedell-Neergaard AS., et al. "Cardiorespiratory fitness and the metabolic syndrome: Roles of inflammation and abdominal obesity". PLoS ONE 13.3 (2018): 1-16.

35. Houzelle, A., et al. "Human skeletal muscle mitochondrial dynamics in relation to oxidative capacity and insulin sensitivity". Diabetologia (2020).

36. Zimmet P., et al. "The metabolic syndrome in children and adolescents". Lancet 369.9579 (2007): 2059-2061.

37. Alberti K G and ZIMMET Z. "Definition, diagnosis and classification of diabetes mellitus and its complications. Part 1 : diagnosis and classification of diabetes mellitus provisional report of a WHO consultation". Diabetic Medicine 15.7 (1998): 539-553.

\section{Assets from publication with us}

- Prompt Acknowledgement after receiving the article

- Thorough Double blinded peer review

- Rapid Publication

- Issue of Publication Certificate

- High visibility of your Published work

Website: www.actascientific.com/

Submit Article: www.actascientific.com/submission.php

Email us: editor@actascientific.com

Contact us: +919182824667 